\title{
Effectiveness of Endoscopic Sclerotherapy with Aluminum Potassium Sulfate and Tannic Acid as a Non-Surgical Treatment for Internal Hemorrhoids
}

\author{
Yuichi Tomiki, Jun Aoki, Shunsuke Motegi, Rina Takahashi, Toshiaki Hagiwara, Yu Okazawa, Kosuke Mizukoshi, Masaya Kawai, \\ Shinya Munakata, Shun Ishiyama, Kiichi Sugimoto and Kazuhiro Sakamoto
}

Department of Coloproctological Surgery, Juntendo University, Faculty of Medicine, Tokyo, Japan

Background/Aims: Sclerotherapy with aluminum potassium sulfate and tannic acid (ALTA) has a potent effect on internal hemorrhoids. In this retrospective study, we compared the effects of endoscopic ALTA therapy and standard ALTA therapy.

Methods: We investigated patients who underwent treatment for internal hemorrhoids at our institution between 2014 and 2016 . They were divided into a standard ALTA group ( $n=33$, treated using proctoscopy) and an endoscopic ALTA group ( $n=48$ ). We compared the clinical findings between the 2 groups.

Results: There were no intergroup differences in background factors. The mean ALTA dose was $21.9 \pm 7.2 \mathrm{~mL}$ and $17.8 \pm 3.4 \mathrm{~mL}$ in the standard and endoscopic ALTA groups, respectively $(p<0.01)$. Adverse events occurred in 4 patients $(12.1 \%)$ from the standard ALTA group and 6 patients (12.5\%) from the endoscopic ALTA group. In both groups, the patients reported good satisfaction with the therapeutic effect at 1 month after the procedure. Hemorrhoids recurred in 2 patients (6.3\%) from the standard ALTA group and 4 patients (8.3\%) from the endoscopic ALTA group.

Conclusions: Endoscopic ALTA sclerotherapy is equivalent to standard ALTA therapy in terms of efficacy, adverse events, and recurrence. Therefore, it is a useful non-surgical option for patients with internal hemorrhoids who prefer a less invasive treatment. Clin Endosc 2019;52:581-587

Key Words: Hemorrhoids; Endoscopy; Therapy; Surgery

\section{INTRODUCTION}

Zione $^{\circledR}$ (J-Dolph Co., Osaka, Japan) is a combination drug containing aluminum potassium sulfate and tannic acid (ALTA) as the active ingredients. It was created by modifying the components of Xiaozhiling, a sclerosant that is approved for injection sclerotherapy of internal hemorrhoids in Chi-

Received: December 21, 2018 Revised: April 13, 2019

Accepted: April 14, 2019

Correspondence: Yuichi Tomiki

Department of Coloproctological Surgery, Juntendo University, Faculty of Medicine, 2-1-1 Hongo, Bunkyo-ku, Tokyo 113-8421, Japan

Tel: +81-3813-3111, Fax: +81-3813-0731, E-mail: tomiki@juntendo.ac.jp ORCID: https://orcid.org/0000-0001-9284-905X

(c) This is an Open Access article distributed under the terms of the Creative Commons Attribution Non-Commercial License (http://creativecommons.org/ licenses/by-nc/3.0) which permits unrestricted non-commercial use, distribution, and reproduction in any medium, provided the original work is properly cited. na. ${ }^{1-3}$ Sclerotherapy has been performed for internal hemorrhoids for many years. ALTA-based sclerotherapy has a potent effect and is routinely used as an option for the non-surgical treatment of hemorrhoids in Japan. ${ }^{4}$

Although non-surgical treatment does not improve internal hemorrhoids to the same extent as surgical treatment, it may be more effective than conservative treatment with suppositories and ointments. Non-surgical treatment is less invasive than surgical treatment and is preferred by many patients. The current non-surgical treatments for internal hemorrhoids include rubber band ligation (RBL), sclerotherapy, and infrared coagulation. These treatments are primarily performed for internal hemorrhoids of Goligher grade II or lower. ${ }^{5,6}$ Among them, RBL is the most widely used method owing to its low cost, simplicity, low incidence of adverse events, and relative efficacy. ${ }^{5,6}$ In addition, $5 \%$ phenol almond oil is a commonly used sclerosant that is effective for bleeding internal hemor- 
rhoids, although not for prolapsed internal hemorrhoids. ${ }^{6,7}$

ALTA therapy was first covered by the Japanese national health insurance system in 2005. It is effective for both bleeding internal hemorrhoids and prolapsed internal hemorrhoids and thus may be a useful alternative to surgery. ${ }^{1-4}$ Although ALTA therapy is considered effective in Asia, it has not yet been adopted elsewhere. $5,7,8$

In ALTA therapy, internal hemorrhoids are subjected to a 4 -step injection procedure that is performed using a proctoscope. ${ }^{1-4}$ With the 4-step injection technique, ALTA is injected into the submucosal layer of internal hemorrhoids. We considered endoscopic submucosal injection to be possible in such cases. Thus, in addition to standard ALTA therapy, we have performed ALTA therapy using an endoscope instead of a proctoscope in some patients. ${ }^{9,10}$

In this study, we retrospectively compared the therapeutic effect between endoscopic ALTA therapy and standard ALTA therapy.

\section{MATERIALS AND METHODS}

Patients who underwent treatment of internal hemorrhoids at our institution between 2014 and 2016 were investigated. The study population consisted of 33 and 48 patients who were treated with standard ALTA therapy and endoscopic ALTA therapy, respectively.

ALTA therapy was indicated for patients with Goligher grade II/III internal hemorrhoids presenting with prolapse and bleeding. Patients with external hemorrhoids or anal polyps were excluded, as were pregnant or lactating women and patients with kidney dysfunction. In patients taking antithrombotic agents, ALTA therapy was performed without discontinuing antithrombotic treatment. ${ }^{11}$ If the symptoms were not relieved by ALTA therapy, conventional hemorrhoidectomy was recommended to the patients.

ALTA therapy was performed using a 4-step injection procedure. In the 1st step, ALTA was injected into the upper pole of the hemorrhoid. In the 2nd and 3rd steps, ALTA was injected into the center of the hemorrhoid. In the 4th step, ALTA was injected into the lower pole of the hemorrhoid. After the injections, the injection sites were massaged (by the physician inserting his/her fingers into the anus of the patient) to diffuse the drug solution. ${ }^{1-4}$

Standard ALTA therapy was carried out in the operation theater. After the application of local anesthesia to the periphery of the anus, with the patient in Sims' position, standard ALTA therapy was performed using a Z-type proctoscope (Arakawa Seisakujo, Tokyo, Japan) and a special needle (ALTA-only needle).
Endoscopic ALTA sclerotherapy was conducted in the endoscopy room after bowel preparation through intestinal lavage. Sedation was induced with pethidine hydrochloride instead of perianal anesthesia, and endoscopic ALTA therapy was conducted using an endoscopic injection needle (25 G, 3 $\mathrm{mm}$ ) by attaching a transparent hood to the end of the scope. According to the 4-step injection technique, the scope was retroflexed in the rectum, and ALTA was injected into the upper pole of the hemorrhoid (1st step). After returning the retroflexed scope to the normal position, ALTA was injected into the center of the hemorrhoid and then into the lower pole (2nd, 3rd, and 4th steps). Before endoscopic ALTA therapy, total colonoscopy was performed to detect other diseases; however, an insurance claim for endoscopy was not submitted. When a lesion required further treatment, endoscopy was promptly performed to confirm the effect of endoscopic ALTA therapy. ${ }^{9,10}$

Analgesia and antibiotics were prescribed after ALTA therapy. Short-term admission (overnight stay) was required in standard ALTA therapy, whereas endoscopic ALTA therapy was performed as a day procedure. Follow-up was performed at 1 week, 1 month, and 1 year after ALTA therapy.

Standard or endoscopic ALTA therapy was selected on the basis of each patient's preference and the attending physician's evaluation after explaining the anesthetic/treatment procedures and admission period to the patients.

We compared the background data of the patients, dose of ALTA administered, complications, adverse events, patients' satisfaction with the therapeutic effect, and frequency of recurrence between the standard ALTA therapy group and the endoscopic ALTA therapy group. The therapeutic effect was evaluated through interviews, digital anal examination, and anoscopy at 1 month after ALTA therapy. A "cure" was defined as the absence of residual symptoms with almost complete resolution of the internal hemorrhoids, whereas "improvement" meant no residual symptoms, with the internal hemorrhoids being reduced in size but still visible on anoscopy. "Failure" was defined as no improvement of symptoms, with the internal hemorrhoids still being visible (no shrinkage). Patient satisfaction was evaluated on a 3-grade scale ("very satisfied", "satisfied", or "dissatisfied") at 1 month after ALTA therapy.

Informed consent was obtained from all patients. This study was conducted after approval by the ethical review board of our hospital (approval no. 488). Results are expressed as means \pm standard deviations, except for the follow-up period (expressed as the median). Statistical analysis was performed using the chi-square test or $t$-test on JMP 9.0 software (SAS Institute Inc., Cary, NC, USA), and $p<0.05$ was considered to indicate significance. 


\section{RESULTS}

\section{Patient profile}

There were no differences in background factors between the standard ALTA therapy group and the endoscopic ALTA therapy group, including age, sex, primary symptoms, grade of internal hemorrhoids, and frequency of antithrombotic treatment (Table 1).

\section{Dose of ALTA}

The mean dose of ALTA in the standard ALTA therapy group and endoscopic ALTA therapy group was $21.9 \pm 7.2 \mathrm{~mL}$ and $17.8 \pm 3.4 \mathrm{~mL}$, respectively $(p<0.01)$.

\section{Therapeutic effect and patient satisfaction}

At 1 month after ALTA therapy, the percentage of patients who achieved a "cure" was higher in the standard ALTA therapy group (30.3\%) than in the endoscopic ALTA therapy group (18.7\%). On the other hand, the percentage of patients exhibiting "improvement" was higher in the endoscopic ALTA therapy group (77.1\%) than in the standard ALTA group (66.7\%). The cases of 1 patient (3.0\%) in the standard ALTA therapy group and 2 patients (4.2\%) in the endoscopic ALTA therapy group were classified as "failure".

The patients' satisfaction with the therapeutic effect at 1 month after the procedure was good in both groups $(>90 \%$ of patients were "very satisfied" or "satisfied" with the result). In the standard ALTA therapy group, a greater proportion of patients were "very satisfied" with the results and a lower proportion of patients were "dissatisfied" compared with the endoscopic ALTA therapy group.

\section{Complications and adverse events}

None of the patients developed intra-procedural complications such as hypotension or bradycardia. Post-procedural adverse events were observed in 4 patients (12.1\%) from the standard ALTA therapy group and 6 patients $(12.5 \%)$ from the endoscopic ALTA therapy group. However, all of these events improved with conservative treatment. The adverse events included mild fever, anal pain, urination disorders, and ulcers. In 1 patient, an asymptomatic ulcer was found during colonoscopy conducted 3 months after endoscopic ALTA therapy for the resection of a colorectal polyp that was detected during the ALTA procedure (Fig. 1). The ulcer was not detected on the anoscopy performed 1 month after ALTA therapy.

\section{Recurrence}

Recurrent hemorrhoids were detected in 2 patients $(6.3 \%)$ from the standard ALTA therapy group and in 4 patients (8.3\%) from the endoscopic ALTA therapy group. One patient (3.0\%) from the standard ALTA therapy group and 3 patients (6.3\%) from the endoscopic ALTA therapy group underwent surgical hemorrhoidectomy within 1 year because they were not satisfied with the therapeutic effects. One patient from the standard ALTA therapy group used suppositories for the treatment of recurrent hemorrhoids. Endoscopic ALTA therapy was repeated in 1 of the 4 patients with recurrent hemorrhoids in the standard group. Recurrence of symptoms was not observed in the other patients who were followed up. The therapeutic effect after 1 year was assessed using anoscopy in 37 patients (45.7\%) and endoscopy in 18 patients (22.2\%). The follow-up of the patients was discontinued at the time of termination of outpatient care on the basis of self-evaluation or

Table 1. The Patients' Characteristics

\begin{tabular}{|c|c|c|c|}
\hline & $\begin{array}{c}\text { Standard ALTA } \\
(n=33)\end{array}$ & $\begin{array}{l}\text { Endoscopic ALTA } \\
\qquad(n=48)\end{array}$ & $p$-value \\
\hline Age & $64.4 \pm 13.9$ & $61.4 \pm 13.1$ & 0.58 \\
\hline \multicolumn{4}{|l|}{ Sex } \\
\hline Male & $27(81.8 \%)$ & $31(64.6 \%)$ & 0.09 \\
\hline Female & $6(18.2 \%)$ & $17(35.4 \%)$ & \\
\hline \multicolumn{4}{|l|}{ Symptoms } \\
\hline Bleeding & $11(33.3 \%)$ & $10(20.9 \%)$ & 0.21 \\
\hline Prolapse & $22(66.7 \%)$ & $38(79.1 \%)$ & \\
\hline \multicolumn{4}{|l|}{ Grade of hemorrhoids ${ }^{\mathrm{a})}$} \\
\hline II & $9(27.3 \%)$ & $9(18.8 \%)$ & 0.36 \\
\hline III & $24(72.7 \%)$ & $39(81.2 \%)$ & \\
\hline Antithrombotic treatment & $6(18.2 \%)$ & $5(10.4 \%)$ & 0.32 \\
\hline
\end{tabular}

ALTA, aluminum potassium sulfate and tannic acid.

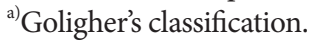


because of death from another disease. The median follow-up period was 498 days in the standard ALTA therapy group and 676 days in the endoscopic ALTA therapy group (Table 2).

\section{Case presentation}

Endoscopic ALTA therapy was performed in a 70-year-old man with prolapsed internal hemorrhoids (Fig. 2A). ALTA containing a low concentration of indigo carmine was injected into 3 hemorrhoids (total volume: $20 \mathrm{~mL}$ ) (Fig. 2B). Then,
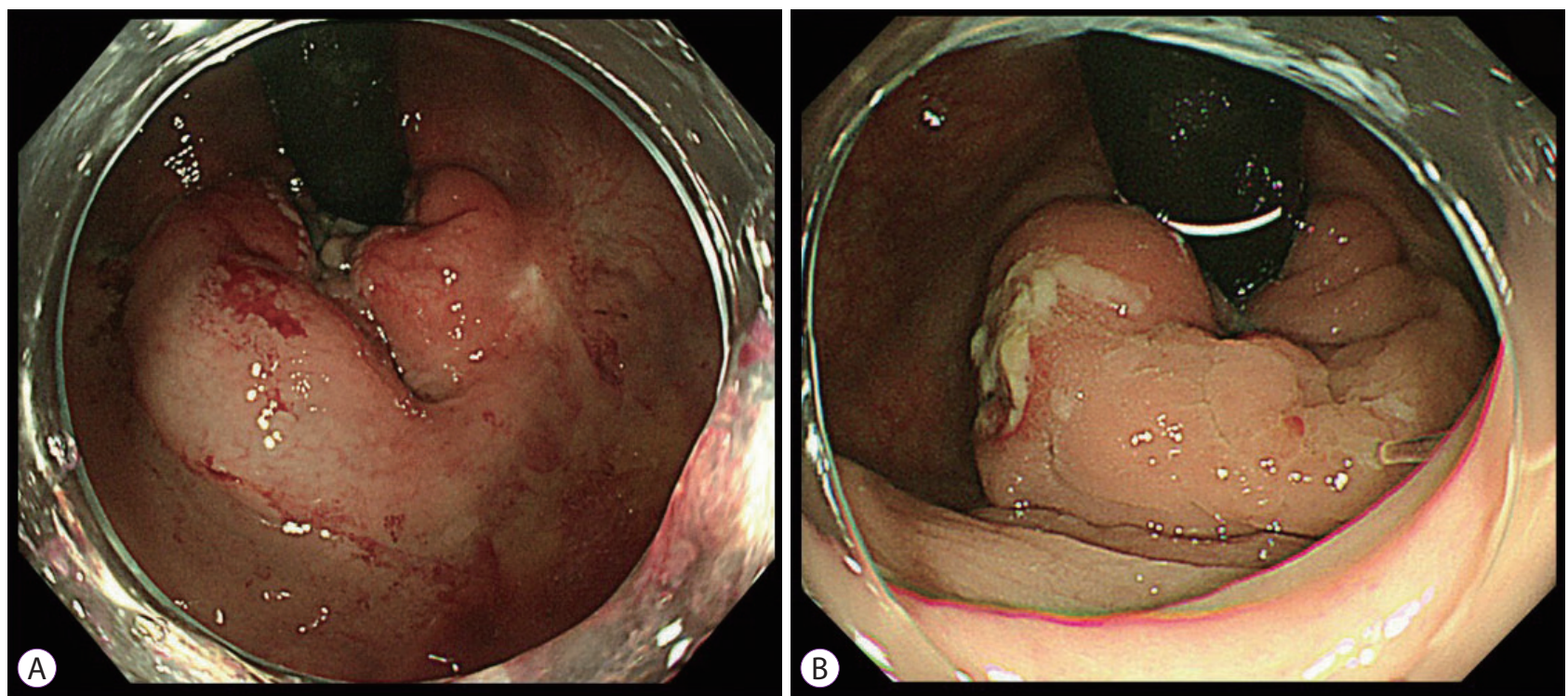

Fig. 1. A rectal ulcer detected after endoscopic aluminum potassium sulfate and tannic acid sclerotherapy. (A) Before treatment. (B) Three months after treatment.

Table 2. Comparison between Standard and Endoscopic Aluminum Potassium Sulfate and Tannic Acid for the Treatment of Internal Hemorrhoids

\begin{tabular}{|c|c|c|c|}
\hline & $\begin{array}{l}\text { Standard ALTA } \\
(n=33)\end{array}$ & $\begin{array}{l}\text { Endoscopic ALTA } \\
\qquad(n=48)\end{array}$ & $p$-value \\
\hline Injection dose $(\mathrm{mL})$ & $21.9 \pm 7.2$ & $17.8 \pm 3.4$ & $<0.01$ \\
\hline \multicolumn{4}{|l|}{ Therapeutic effects } \\
\hline Cure & $10(30.3 \%)$ & $9(18.7 \%)$ & 0.22 \\
\hline Improvement & $22(66.7 \%)$ & $37(77.1 \%)$ & 0.30 \\
\hline Failure & $1(3.0 \%)$ & $2(4.2 \%)$ & 0.79 \\
\hline \multicolumn{4}{|l|}{ Patient satisfaction } \\
\hline Very satisfied & $20(60.6 \%)$ & $20(41.7 \%)$ & 0.09 \\
\hline Satisfied & $11(33.3 \%)$ & $24(50.0 \%)$ & 0.14 \\
\hline Dissatisfied & $2(6.1 \%)$ & $4(8.3 \%)$ & 0.70 \\
\hline $\begin{array}{l}\text { Adverse events } \\
\text { (including multiple events) }\end{array}$ & $4(12.1 \%)$ & $6(12.5 \%)$ & 0.96 \\
\hline Low-grade fever & $1(3.0 \%)$ & $1(2.1 \%)$ & 0.78 \\
\hline Anal pain & $2(6.1 \%)$ & $4(8.3 \%)$ & 0.70 \\
\hline Urinary retention & $1(3.0 \%)$ & $1(2.1 \%)$ & 0.78 \\
\hline Rectal ulcer & $1(3.0 \%)$ & $1(2.1 \%)$ & 0.78 \\
\hline Recurrence & $2(6.1 \%)$ & $4(8.3 \%)$ & 0.70 \\
\hline Within 1 yr & $1(3.0 \%)$ & $3(6.3 \%)$ & 0.51 \\
\hline Follow-up period (days) & $498(28-1,485)$ & $676(28-1,124)$ & \\
\hline
\end{tabular}

ALTA, aluminum potassium sulfate and tannic acid. 
diffusion of the drug solution was promoted by massaging the injection sites (Fig. 2C). Endoscopy at 8 months after treatment showed reduction in the size of the hemorrhoids and no prolapse (Fig. 2D). Recurrence was not detected during the post-procedural follow-up period of 2 years.

\section{DISCUSSION}

ALTA sclerotherapy was first covered by the Japanese national health insurance system as a non-surgical treatment for hemorrhoids in 2005. Since then, it has been performed for many patients in Japan. ${ }^{4}$ With respect to the mechanism of action of ALTA, it rapidly shows a hemostatic effect by reducing blood flow in the treated hemorrhoids. ALTA also induces inflammation and/or fibrosis in the tissue surrounding the hemorrhoids, which prevents prolapse. ${ }^{1-4}$ The merits of ALTA sclerotherapy compared with surgery include minimal invasiveness, mild post-procedure pain, slight bleeding, and shortterm admission (it can be performed as a day procedure). ${ }^{4,8}$ On the other hand, there is a higher recurrence rate than after surgical hemorrhoidectomy. However, many patients with internal hemorrhoids prefer a day procedure or minimally invasive treatment involving short-term admission over surgery, even if the recurrence rate is slightly higher, and ALTA therapy is a useful option in such cases. Furthermore, ALTA therapy can be performed without discontinuing antithrombotic treatment. ${ }^{11}$ Even if hemorrhoids recur, it is possible to repeat ALTA therapy.

Treatment of internal hemorrhoids using an endoscope
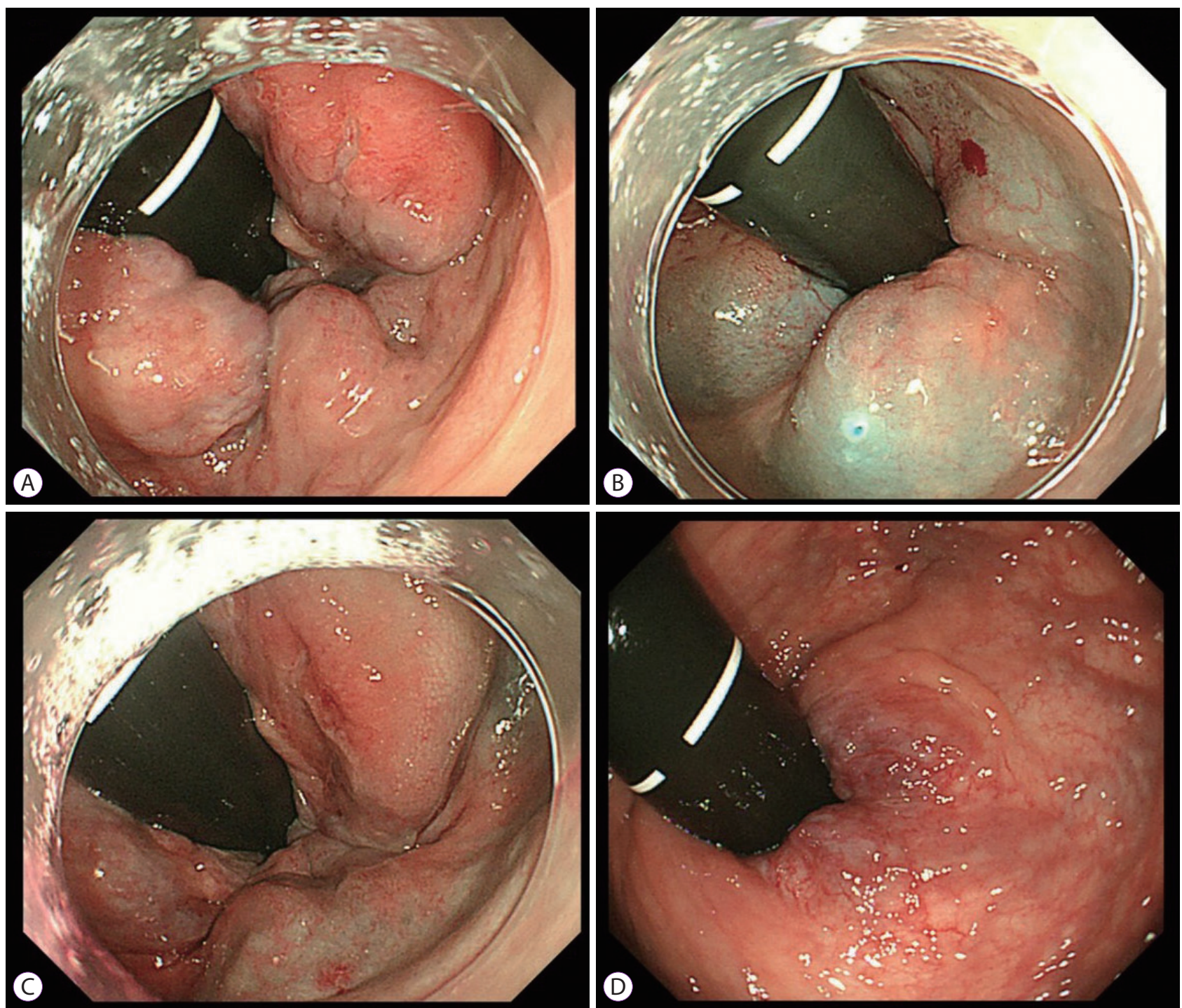

Fig. 2. Endoscopic aluminum potassium sulfate and tannic acid (ALTA) sclerotherapy for Goligher grade III prolapse. (A) Before treatment. (B) After ALTA injection. (C) After finger massage. (D) At 8 months after treatment. 
instead of a proctoscope is not a new procedure, and endoscopes have also been used for sclerotherapy, RBL, and infrared coagulation. ${ }^{12-14}$ In endoscopic ALTA sclerotherapy, a colonoscope and endoscopic injection needle are used as a long proctoscope and long needle, respectively; thus, this procedure is similar to the standard ALTA therapy. As ALTA is mainly injected into the submucosal layer, using an endoscope may help ensure that the injection site is correct. According to a previous study, a 3-mm-long endoscopic injection needle is unlikely to reach a depth immediately above the muscle layer. Therefore, although the procedure is safe, the drug solution may not penetrate the region immediately above the muscle layer. ${ }^{13}$ However, even if the drug solution is only injected into the superficial part of the submucosal layer, it can be spread to the region immediately above the muscle layer by massaging the injection site after the procedure. Thus, use of an endoscopic needle to inject ALTA may be acceptable. Furthermore, the drug solution can be accurately injected into the upper pole of a hemorrhoid by reversing the endoscope, which is advantageous. As demonstrated by the transition from laparotomy to laparoscopic surgery over the years, the magnifying effect of endoscopy and the ability to share the vision of the operating field may also be very useful for anal procedures. However, Japanese proctologists consider tactile sensation to be important during digital palpation and primarily select treatments involving the use of a conventional proctoscope for internal hemorrhoids, which means endoscopic treatment is still not widely accepted.

In the current study, it is possible that more patients underwent endoscopic ALTA therapy rather than standard ALTA therapy because the endoscopic method is a day procedure, thereby being more attractive to the patients. Although standard ALTA therapy can also be performed as a day procedure, it was carried out in the operating theater in the present study and short-term admission (overnight stay) was required. Furthermore, the endoscopic ALTA group had a percentage of female patients, possibly because the endoscopic method reduces the embarrassment associated with the treatment.

ALTA was injected at a volume of $7-10 \mathrm{~mL}$ per internal hemorrhoid, although the dose differed between the 2 groups. Because endoscopic ALTA therapy has not been recommended in ALTA sclerotherapy workshops, we performed it carefully and used a similar bowel preparation protocol to that used for colonoscopy in order to prevent accidents. In addition, we covered the tip of the endoscope with a transparent hood to add anoscopic functionality and confirmed that ALTA was injected into the submucosal layer while observing distension at the injection site on a monitor. We used a lower ALTA dose in this series as a precaution; however, it would be preferable to increase the ALTA dose in the future while monitoring the patients for adverse events.

Although the therapeutic effect was evaluated for a short period of 1 month after ALTA therapy, >95\% of the patients in each group showed "improvement" or "cure". Consistent with this therapeutic effect, the patients were generally satisfied with the results of ALTA sclerotherapy after 1 month. In addition to 19 patients who achieved "cure", 21 patients who demonstrated "improvement" were "very satisfied" with the treatment. This suggests that improvement of symptoms without complete cure was sufficient for patients to become "very satisfied". On the other hand, although the treatment failed in only 3 patients, 6 patients were "dissatisfied" with the therapeutic effect. This suggests that some patients found that their symptoms did not improve as much as expected. In those patients who were not satisfied with the outcome, ALTA therapy did not result in disappearance of external hemorrhoids or anal skin tags. Thus, those who were not satisfied with the result may have been disappointed that ALTA therapy was not effective in eliminating external hemorrhoids or skin tags, as expected. In the future, the combination of excision and ALTA therapy may be indicated for patients with internal hemorrhoids who also have external hemorrhoids, anal polyps, or anal skin tags. ${ }^{8,15}$

Adverse events were observed in approximately $12 \%$ of the patients in both the standard and endoscopic ALTA therapy groups. The most frequent adverse event was anal pain; however, anal discomfort is frequent after sclerotherapy and it is unclear whether this should be considered anal pain. Oral analgesics relieved the pain within a few days. However, painless ulceration was noted in some patients. This may have been associated with the 1st step of injection into the submucosal layer at the upper pole of the hemorrhoid. When RBL is performed, the hemorrhoid is ligated and allowed to fall off, leading to superficial ulceration. ${ }^{16}$ However, the ALTA-related ulcers remained at 3 months after sclerotherapy, suggesting the need for careful post-procedural follow-up in such cases. Although anoscopy was performed at 1 month after ALTA therapy, ulcer formation might have been detected more frequently if endoscopy was conducted for all patients after the procedure. Because total colonoscopy was performed before ALTA therapy, re-examination after a short period would be burdensome for patients in the endoscopic ALTA group. However, recording the findings before and after ALTA therapy is important. As the subsequent course was observed using endoscopy in only $22.2 \%$ of all patients, it may be necessary to adopt other measures, such as endoscopy with only enema preparation.

The recurrence rate was $8.3 \%$ in the endoscopic ALTA therapy group, which did not differ significantly from that in the standard ALTA therapy group (6.1\%) or the rates reported 
previously. This recurrence rate may have been related to the lower ALTA dose used in the endoscopic group. In the future, endoscopic ALTA therapy with a higher dose should be performed while taking care to avoid adverse events. Further follow-up may be necessary to examine the long-term outcomes of such treatment. ${ }^{17}$

This study has several limitations. It was a retrospective, non-randomized, single-center study with a small sample size. Moreover, despite the widespread use of ALTA in Japan, only a limited number of institutions perform endoscopic ALTA sclerotherapy. Therefore, the efficacy and safety of endoscopic ALTA sclerotherapy should be investigated by performing a multicenter study in the future.

For patients who wish to obtain complete cure of hemorrhoids, surgical hemorrhoidectomy is the most effective treatment. However, ALTA therapy is sufficient for those who want to achieve improvement of their symptoms. In particular, endoscopic ALTA therapy does not require perianal anesthesia and may be appropriate for patients without concomitant lesions, such as external hemorrhoids.

There were no significant differences between endoscopic ALTA therapy and standard ALTA therapy in terms of efficacy, adverse events, and recurrence. Therefore, endoscopic ALTA sclerotherapy is an effective and minimally invasive non-surgical treatment option for patients with internal hemorrhoids.

\section{Conflicts of Interest \\ The authors have no financial conflicts of interest. \\ Author Contributions \\ Conceptualization: Yuichi Tomiki, Jun Aoki \\ Data curation: YT, JA, Shunsuke Motegi, Rina Takahashi, Toshiaki Hagi- wara, Masaya Kawai \\ Investigation: Shun Ishiyama, Kiichi Sugimoto \\ Resources: Yu Okazawa, Kosuke Mizukoshi, MK \\ Writing-original draft: YT \\ Writing-review\&editing: YT, Shinya Munakata, Kazuhiro Sakamoto}

\section{REFERENCES}

1. Hachiro Y, Kunimoto M, Abe T, Kitada M, Ebisawa Y. Aluminum potassium sulfate and tannic acid (ALTA) injection as the mainstay of treatment for internal hemorrhoids. Surg Today 2011;41:806-809.

2. Miyamoto $\mathrm{H}$, Asanoma M, Miyamoto $\mathrm{H}$, Shimada M. ALTA injection sclerosing therapy:non-excisional treatment of internal hemorrhoids. Hepatogastroenterology 2012;59:77-80.

3. Tokunaga Y, Sasaki H. Impact of less invasive treatments including sclerotherapy with a new agent and hemorrhoidopexy for prolapsing internal hemorrhoids. Int Surg 2013;98:210-213.

4. Miyamoto H, Hada T, Ishiyama G, Ono Y, Watanabe H. Aluminum potassium sulfate and tannic acid sclerotherapy for Goligher Grades II and III hemorrhoids: results from a multicenter study. World J Hepatol 2016;8:844-849.

5. Davis BR, Lee-Kong SA, Migaly J, Feingold DL, Steele SR. The American Society of Colon and Rectal Surgeons Clinical Practice Guidelines for the management of hemorrhoids. Dis Colon Rectum 2018;61:284292.

6. Brown SR. Haemorrhoids: an update on management. Ther Adv Chronic Dis 2017;8:141-147.

7. Yano T, Yano K. Comparison of injection sclerotherapy between 5\% phenol in almond oil and aluminum potassium sulfate and tannic acid for grade 3 hemorrhoids. Ann Coloproctol 2015;31:103-105.

8. Lim SW. Aluminum potassium sulfate and tannic acid injection for hemorrhoids. J Korean Soc Coloproctol 2012;28:73-77.

9. Tomiki Y, Ono S, Aoki J, Takahashi R, Sakamoto K. Endoscopic sclerotherapy with aluminum potassium sulfate and tannic acid for internal hemorrhoids. Endoscopy 2014;46 Suppl 1 UCTN:E114.

10. Tomiki Y, Ono S, Aoki J, et al. Treatment of internal hemorrhoids by endoscopic sclerotherapy with aluminum potassium sulfate and tannic acid. Diagn Ther Endosc 2015;2015:517690.

11. Yano T, Nogaki T, Asano M, Tanaka S, Kawakami K, Matsuda Y. Outcomes of case-matched injection sclerotherapy with a new agent for hemorrhoids in patients treated with or without blood thinners. Surg Today 2013;43:854-858.

12. ASGE Technology Committee, Siddiqui UD, Barth BA, et al. Devices for the endoscopic treatment of hemorrhoids. Gastrointest Endosc 2014;79:8-14.

13. Zhang T, Xu LJ, Xiang J, et al. Cap-assisted endoscopic sclerotherapy for hemorrhoids: methods, feasibility and efficacy. World J Gastrointest Endosc 2015;7:1334-1340.

14. Tomiki Y, Higashiyama A, Okada T, et al. Evaluation of endoscopic hemorrhoidal ligation in 119 patients. Dig Endosc 2003;15:30-34.

15. Abe T, Hachiro Y, Ebisawa Y, Hishiyama H, Kunimoto M. Distal hemorrhoidectomy with ALTA injection: a new method for hemorrhoid surgery. Int Surg 2014;99:295-298.

16. Albuquerque A. Rubber band ligation of hemorrhoids: a guide for complications. World J Gastrointest Surg 2016;8:614-620.

17. Yano T, Asano M, Tanaka S, Oda N, Matsuda Y. Prospective study comparing the new sclerotherapy and hemorrhoidectomy in terms of therapeutic outcomes at 4 years after the treatment. Surg Today 2014;44:449453. 